\title{
COMPOSICIÓN QUÍMICA Y PRODUCCIÓN DE GAS IN VITRO DE DIETAS CON VAINAS DE ACACIA FARNESIANA
}

\author{
CHEMICAL COMPOSITION AND IN VITRO GAS PRODUCTION OF DIETS \\ WITH ACACIA FARNESIANA PODS
}

\begin{abstract}
Velázquez, A.J. ${ }^{1}$, González Ronquillo, M. ${ }^{1 *}$, Bórquez, J. ${ }^{1}$, Domínguez, I.A. ${ }^{1}$ y Perezgrovas, R. ${ }^{2}$
'Departamento de Nutrición Animal. Facultad de Medicina Veterinaria y Zootecnia. Universidad Autónoma del Estado de México. Instituto Literario 100 Ote. Toluca. México. *mrg@uaemex.mx

${ }^{2}$ Instituto de Estudios Indígenas. Universidad Autónoma de Chiapas. México.
\end{abstract}

\section{PALABRAS CLAVE ADICIONALES}

Escasez de alimentos. Leguminosa arbustiva. Sistemas de producción. Taninos.

\section{RESUMEN}

Se valoró el efecto de la inclusión de vainas de Acacia farnesiana en dietas para corderos. Los niveles de $A$. farnesiana en los tratamientos fueron $0,100,200,300$ y $400 \mathrm{~g} / \mathrm{kg}$ MS en dietas isoprotéicas para corderos en crecimiento que incluyeron rastrojo de maíz, harina de soya, salvado de trigo y mezcla de minerales. Las vainas aportan una importante cantidad de proteína (115 $\mathrm{g} / \mathrm{kg})$; de taninos $(7,8 \mathrm{~g} / \mathrm{kg})$. La producción de gas in vitro disminuyó $(p<0,05)$ con la inclusión de 400 $\mathrm{g} / \mathrm{kg}(304,45 \mathrm{ml} / \mathrm{g} \mathrm{MS}) ;$ no obstante, fue similar a $300 \mathrm{~g} / \mathrm{kg}(317,88 \mathrm{ml} / \mathrm{g} \mathrm{MS})$. La desaparición de la materia seca in vitro fue semejante en 0 y $100 \mathrm{~g} / \mathrm{kg}$ con 70,14 y $68,73 \mathrm{mg} / 100 \mathrm{mg}$ MS respectivamente; no obstante, ambas fueron diferentes al resto de los tratamientos. La producción de gas relativa fue similar entre $200 \mathrm{a} 400 \mathrm{~g} / \mathrm{kg}$, aunque diferentes a la dieta control. Inclusión de $300 \mathrm{~g} / \mathrm{kg}$ MS de vainas de $A$. farnesiana impacta negativamente en la producción de gas y energía metabolizable, y a partir de $200 \mathrm{~g} / \mathrm{kg}$ MS afecta la desaparición de la materia seca y la producción de gas relativa in vitro lo cual podría limitar su uso y aprovechamiento para la alimentación de rumiantes.

\section{SUMMARY}

The inclusion of Acacia farnesiana pods into lamb diets was evaluated by means of analyzing

\section{AdDitional KEYWORDS}

Food scarcity. Leguminous shrub. Production systems. Tannins.

its chemical composition, tannin content and in vitro gas production, dry matter disappearance and metabolizable energy. The treatments with different inclusion levels of $A$. farnesiana pods were $0,100,200,300$ and $400 \mathrm{~g} / \mathrm{kg} \mathrm{DM}$, within isoprotein diets for lambs which included ground maize fodder, soybean meal, wheat bran and mineral mix. The $A$. farnesiana pods contributed with an important amount of protein $(115 \mathrm{~g} / \mathrm{kg})$, and tannins $(7.8 \mathrm{~g} / \mathrm{kg})$. In vitro gas production decreased $(p<0.05)$ with the inclusion of $400 \mathrm{~g} / \mathrm{kg}$ DM $(304.45 \mathrm{ml} / \mathrm{g} \mathrm{DM})$; nonetheless, its production was similar to $300 \mathrm{~g} / \mathrm{kg}(317.88 \mathrm{ml} / \mathrm{g} \mathrm{DM})$. In vitro dry matter disappearance (IVDMD) in 0 and $100 \mathrm{~g} /$ $\mathrm{kg}$ was similar between them with 70.14 and 68.73 $\mathrm{mg} / 100 \mathrm{mg}$ DM respectively; however, they were different than the rest of the treatments. Relative gas production was similar between 200 to $400 \mathrm{~g} /$ $\mathrm{kg}$, nevertheless it was different to control diets. It is concluded that the inclusion of $300 \mathrm{~g} / \mathrm{kg} \mathrm{DM}$ of A. farnesiana pods has a negative impact over in vitro gas production and metabolizable energy, and over $200 \mathrm{~g} / \mathrm{kg}$ DM affect the dry matter disappearance and relative in vitro gas production, which limit its use for feeding ruminants.

\section{INTRODUCCIÓN}

En sistemas campesinos de México, la 
producción animal es afectada por la escasez de alimentos y recursos económicos, lo que limita su rentabilidad (Arriaga-Jordán et al., 2001). En ese sentido, los árboles y arbustos forrajeros, como las acacias, cobran relevancia considerando que son cosmopolitas; sin embargo, los taninos que contienen pueden restringir, en términos de nutrición y eficiencia productiva, su aprovechamiento (Seigler, 2003; Velázquez et al., 2011), debido a su reacción con polisacáridos, proteínas, etc., y complejos que pueden ser precipitados, reduciendo la fracción degrabable y su tasa de degradación al inhibir el crecimiento bacteriano y la actividad enzimática (McSweeney et al., 2001; Bennie et al., 2002; Min et al., 2003; Frutos et al., 2004).

La Acacia farnesiana es una leguminosa arbustiva perenne con potencial forrajero que puede ser aprovechada como fuente de proteína para dietas de bajo costo (Perezgrovas, 1999; Velázquez et al., 2011). En Chiapas, México, sus vainas constituyen una alternativa poco estudiada y su investigación, en beneficio de los pequeños rumiantes, es importante debido a la escasa disponibilidad de nutrientes de los sistemas de producción campesino e indígena, particularmente en el periodo de estiaje. El objetivo del presente estudio fue determinar el efecto de mezclas nutricionales con diferentes niveles de vainas de $A$. farnesiana sobre la composición química, producción de gas in vitro, desaparición de la materia seca y energía metabolizable en ovinos.

\section{MATERIAL Y MÉTODOS}

\section{Material Vegetativo}

Fueron recolectadas a granel vainas secas de $A$. farnesiana, cortadas y acarreadas diariamente, a partir de febrero hasta mayo de 2006 en predios rurales del municipio de Teopisca, Chiapas, ubicados en la región II Altos de Chiapas a $16^{\circ} 15^{\prime}$ y $17^{\circ} 17^{\prime}$ $\mathrm{Ny} 91^{\circ} 32^{\prime}$ y $93^{\circ} 35^{\prime} \mathrm{O}$ a una altitud de 1200 a $2400 \mathrm{~m}$ sobre el nivel del mar. Esta región tiene clima templado subhúmedo $(\mathrm{CW} 2)(\mathrm{W})$, con época de lluvias de junio a noviembre y de secas de diciembre a mayo (Parra, 1989). Se molieron en un molino de martillos marca Dumex, modelo TH4000 y se guardaron en bolsas de plástico para posteriores análisis. Las vainas molidas se incluyeron en cinco dietas isoprotéicas para corderos en crecimiento (NRC, 2007) a diferentes niveles de inclusión: 0, 100, 200, 300 y $400 \mathrm{~g} / \mathrm{kg} \mathrm{MS}$, mezcladas con rastrojo de maíz, harina de soya, salvado de trigo y minerales, que se utilizan tradicionalmente en la región. Se tomaron muestras por tratamiento para análisis químico y se molieron en molino Thomas-Wiley con criba de $2 \mathrm{~mm}$.

\section{COMPOSICIÓNQUÍMICA}

Se estudió la composición química de ingredientes y tratamientos utilizando el procedimiento 940.25 de AOAC (1990) para determinar proteína bruta(PB), 920.39B para extracto etéreo (EE), 938.08 para cenizas (CEN) y 952.08 para materia seca (MS). El análisis de fibra detergente neutra (FDN), fibra detergente ácida (FDA) y lignina detergente ácida (LDA) fueron realizados según Van Soest (1994) usando 0,5 g de muestra en bolsas de filtro (Ankom Corp. F57) y colocadas en el analizador de fibras (Ankom 200/220) utilizando la solución química premezclada para FDN y FDA (Ankom Tecnología, FND20C y FAD20C). La LDA se estimó tratando la FDA con $72 \%$ de ácido sulfúrico. Los valores de FDN y FDA se expresan con cenizas residuales. El valor de energía metabolizable (EM, MJ/Kg MS) se obtuvo según Menke y Steingass (1988). El contenido de taninos (TAN) se estimó por el método de las proantocianidinas según Makkar(2003), utilizando butanol/ $\mathrm{HCl}(95: 5$ $\mathrm{v} / \mathrm{v})$ y sulfato férrico (20 g/12 M HCl); la absorbancia se midió contra un blanco a 550 nm.

\section{Produccióndegas ydesapaRICIÓNDELA MATERIA SECA}

Se estudió la producción de gas in vitro 


\section{PRODUCCIÓN DE GAS IN VITRO CON INCLUSIÓN DE ACACIA FARNESIANA}

(PGIV, $\mathrm{ml} / \mathrm{g}$ MS), desaparición de la materia seca in vitro (DMSIV, $\mathrm{mg} / 100 \mathrm{mg}$ ), tasa de degradación (TD), producción de gas relativa (PGR, ml/g MS desaparecida $96 \mathrm{~h}$ ) y contenido de energía metabolizable in vitro (EMIV, MJ/kg). La PGIV se analizó según la metodología de Theodorou et al. (1994), utilizando $800 \mathrm{mg}$ MS por tratamiento, estándares y blancos, en frascos de $125 \mathrm{ml}$ a los que se agregó $100 \mathrm{ml}$ de líquido ruminal $(1: 9 \mathrm{v} / \mathrm{v}) \mathrm{y}$ medio de incubación (solución búfer, resazurina y cisteína diluida en agua destilada). Los frascos se sumergieron en baño de agua a $39^{\circ} \mathrm{C}$ leyendo y registrando el gas producido $\left(\mathrm{PSI} / \mathrm{cm}^{3}\right)$, a las $3,6,9,12$, $18,24,36,48,72$ y 96 h de incubación. El líquido ruminal se obtuvo de dos bovinos canulados en rumen alimentados, en confinamiento, con heno de alfalfa $(3,2 \mathrm{~kg})$ mezclado con rastrojo de maíz $(5,4 \mathrm{~kg})$ y $2,5 \mathrm{~kg}$ de concentrado comercial (12\% PB, 11,5 MJ EM) por día. El fluido ruminal se obtuvo en la mañana con los animales en ayuno a través de la fístula ruminal. El líquido se colocó en dos termos, separando por animal y llevados de inmediato al laboratorio donde se filtró a través de dos capas de gasa y finalmente se mezcló ambos líquidos. Al finalizar el tiempo de incubación el contenido de cada frasco fue filtrado en bolsas de nylon con poro de $45 \mu \mathrm{m}$, el residuo se secó a $70^{\circ} \mathrm{C}$ durante $24 \mathrm{~h}$ para calcular la DMSIV.

\section{CÁlCULOS Y ANÁlisIS ESTADístico}

La formulación de los tratamientos, incluyendo calcio y fósforo, se calculó aplicando las necesidades nutricionales para ovinos en crecimiento propuestos por la NRC (2007). La PGIV y la TD se determinaron a partir de los volúmenes de gas registrados en las lecturas y analizados con el modelo de France et al. (1993):

$$
y=A\left\{1-\exp \left[-b^{(t-T)}-c^{\left(V_{t}-V_{T}\right)}\right]\right\}
$$

Donde:

y= producción de gas acumulada (ml); $\mathrm{t}=$ tiempo de incubación (h);

$\mathrm{A}=$ asíntota de la curva (producción total de gas; $\mathrm{ml})$;

$\mathrm{b}=$ tasa constante de producción de gas $\left(\mathrm{h}^{-1}\right)$;

$\mathrm{T}=$ tiempo lag (h);

$\mathrm{C}=$ tasa constante de producción de gas $\left(\mathrm{h}^{-1 / 2}\right)$.

La TD $\left(\mu, h^{-1}\right)$ se calculó con la ecuación:

$\mu=b+c /(2 \sqrt{ } t): 1 \geq T$

Que postula que la tasa de degradación fraccional $\left(\mu, h^{-1}\right)$ no es constante y varía con el tiempo a lo largo del periodo de fermentación. La incubación se validó contrastando la PGIV de las muestras contra la producción de cada estándar $(0,9<\mathrm{Fstd}<1,1)$. La tasa de producción de gas relativa (PGR, ml gas/g MS desaparecida a las 96 h) se calculó de los volúmenes de gas registrados según González Ronquillo et al. (1998). La EMIV se estimó con la metodología propuesta por Menke y Steingass (1988):

$\mathrm{EM}=\mathrm{MJ} / \mathrm{kg} \mathrm{MS}=3,16+0,0695 \mathrm{PG}+0,00073 \mathrm{PG}^{2}+$ $0,00732 X P+0,02052 X L$

Donde:

$P G=$ volumen de producción de gas 24 h después de la incubación ( $\mathrm{ml} \mathrm{gas/mg} \mathrm{MS);}$

$\mathrm{XP}=$ proteína bruta;

$X L=$ grasa $(g / k g M S)$.

Se consideró un diseño experimental completamente al azar con cinco tratamientos y tres repeticiones. Se hizo un análisis de varianza y en las variables con significancia $(p<0,05)$ se realizó una comparación de medias por la prueba de Tukey. Además se hizo un análisis de regresión para establecer las ecuaciones, los coeficientes de determinación $\left(\mathrm{R}^{2}\right)$ para cada una de las variables y la significancia de la prueba (Steel y Torrie, 1980), se establecieron líneas de tendencia polinomiales de segundo orden. Los datos fueron procesados con la paquetería Minitab V. 10.51. 
VELÁZQUEZ, GONZÁLEZRONQUILLO, BÓRQUEZ, DOMÍNGUEZY PEREZGROVAS

Tabla I. Composición química ( $\mathrm{g} / \mathrm{kg} \mathrm{MS}$ ) de los ingredientes utilizados en la formulación de raciones. (Chemical composition $(\mathrm{g} / \mathrm{kg} \mathrm{DM})$ of the ingredients used in the formulation ration).

\begin{tabular}{lcccccccc}
\hline Concepto & MS & MO & PB & FDN & FDA & LDA & EE & TAN \\
\hline Vainas de A. farnesiana & 896 & 962 & 115 & 476 & 373 & 134 & 18 & 7,8 \\
Rastrojo de maíz & 898 & 973 & 57 & 659 & 397 & 114 & 27 & - \\
Harina de soya & 901 & 979 & 477 & 176 & 74 & 59 & 21 & - \\
Salvado de trigo & 893 & 961 & 157 & 509 & 188 & 157 & 39 & - \\
\hline
\end{tabular}

$\mathrm{MS}=$ materia seca expresada sobre materia fresca; $\mathrm{MO}=$ materia orgánica; $\mathrm{PB}=$ proteína bruta; $\mathrm{FDN}=$ fibra detergente neutra; $F D A=$ fibra detergente ácida; $L D A=$ lignina detergente ácida; $C E N=$ cenizas; $E E=$ extracto etéreo; $\mathrm{TAN}=$ taninos.

\section{RESULTADOSYDISCUSIÓN}

Los resultados muestran que las vainas de A. farnesiana contienen $115 \mathrm{~g} / \mathrm{kg}$ de proteína bruta (PB), los cuales son superiores al rastrojo de maíz, pero inferiores al salvado y la harina de soya, en cuanto al contenido de fibra detergente neutra (FDN), la acacia fue similar al salvado, e inferior al rastrojo de maíz, los taninos (TAN) sólo se determinaron en A. farnesiana, $7,8 \mathrm{~g} / \mathrm{kg}$ TAN (tabla I) al considerarla como única fuente de taninos en la ración. Reportes de literatura sobre TAN en vainas de $A$. farnesiana mostraron una variación comparada con los resultados de este estudio, Benny et al. (2001) encontraron $194 \mathrm{~g} / \mathrm{kg}$ en vainas $\sin$ semilla y $54 \mathrm{~g} / \mathrm{kg}$ en semillas provenientes del bajío en la región central de México. Monforte-Briseño et al. (2005) encontraron una concentración de $335 \mathrm{~g} / \mathrm{kg}$ de TAN en la región central y norte de Yucatán, México mientras que Velázquez et al. (2005) encontraron $17,6 \mathrm{~g} / \mathrm{kg}$ MS en vainas recolectadas en Chiapas. Es probable que estas diferencias se deban al método de extracción de taninos, ya que Benny et al. (2001) utilizó la metodología FAS y Velázquez et al. (2005) el de vainillina- $\mathrm{HCl}$ considerados menos específicos que la metodología seguida en el presente estudio (Makkar, 2003).

Por otro lado, debe tenerse presente que la región de origen del material vegetativo puede tener efecto en la variación de TAN y PB de las acacias (Frutos et al., 2004; Salem, 2005), tanto como la parte de la planta que se estudie. Purata et al. (1999) encontraron $121 \mathrm{~g} / \mathrm{kg}$ en vainas de $A$. pennatula recolectadas en el área de Veracruz, México; mientras que McSweeney et al. (2005) presentaron un rango de 98 a $180 \mathrm{~g} / \mathrm{kg}$ de TAN en hojas de $A$. angustissima recolectadas en México y América Central. En contraste Aganga y Adogla (1999) y Aganga et al. (2000) reportaron $16 \mathrm{~g} / \mathrm{kg}$ de TAN en semillas de $A$. gerrardii y $68,2 \mathrm{~g} / \mathrm{kg}$ en $A$. sieberana así como un amplio rango en $\mathrm{PB}$ con $125 \mathrm{~g} / \mathrm{kg}$ en $A$. robusta a $218 \mathrm{~g} / \mathrm{kg}$ en $A$. erubescens. En cuanto la composición química del rastrojo de maíz, la harina de soya y el salvado, se encuentran dentro del rango que cita la literatura (Ewin, 1997; NRC, 2007).

La tabla II presenta el contenido nutrimental de los tratamientos, se observa una relación directamente proporcional entre el nivel de inclusión de vainas en las dietas y el contenido de taninos. El contenido de PB de los tratamientos estuvo en un rango de $113,1-118,9 \mathrm{~g} / \mathrm{kg}$ y la EM varió de 8,13 a $9,39 \mathrm{Mj} / \mathrm{kg}$. El contenido de fibra de las dietas fue suministrado principalmente por el rastrojo de maíz y las vainas, cuyos valores son similares, en contraste a lo reportado por Rubanza et al. (2005) en hojas de $A$. nilótica que contiene $222 \mathrm{~g} / \mathrm{kg}$ de FDN; pero, similares con la $A$. polyacantha con $505 \mathrm{~g} / \mathrm{kg}$. También se observó que las vainas de $A$. farnesiana contribuyeron de ma- 
Tabla II. Ingredientes y composición química $(\mathrm{g} / \mathrm{kg} \mathrm{MS)}$ de tratamientos con diferentes niveles de inclusión de vainas de A farnesiana. (Ingredients and chemical compostition (g/kg DM) of the treatments with different inclusion levels of $A$. farnesiana pods).

\begin{tabular}{lccccc}
\hline Vainas de A. farnesiana g/kg & 0,0 & 100 & 200 & 300 & 400 \\
\hline Rastrojo de maíz & 795 & 710 & 624 & 538 & 450 \\
Harina de soya & 130 & 115 & 101 & 87 & 75 \\
Salvado de trigo & 50 & 50 & 50 & 50 & 50 \\
Minerales $^{1}$ & 25 & 25 & 25 & 25 & 25 \\
Composición química de tratamientos & & & & & \\
Materia seca & 910 & 903 & 914 & 914 & 917 \\
Materia orgánica & 947 & 944 & 943 & 944 & 940 \\
Proteína bruta & 117 & 119 & 113 & 113 & 116 \\
Fibra detergente neutra & 540 & 507 & 562 & 561 & 543 \\
Energía metabolizable (MJ/kg) & 9,39 & 8,93 & 9,09 & 8,44 & 8,13 \\
Calcio & 6,5 & 6,5 & 6,5 & 6,5 & 6,5 \\
Fósforo & 4,5 & 4,5 & 4,5 & 4,5 & 4,5 \\
Taninos & 0,05 & 1,2 & 2,2 & 2,9 & 3,6 \\
\hline
\end{tabular}

${ }^{1}$ Minerales incluidos: $\mathrm{Ca}=15 \% ; \mathrm{P}=7 \% ; \mathrm{Mg}=0,25 \% ; \mathrm{Zn}=0,45 \% ; \mathrm{Cu}=0,05 \% ; \mathrm{Co}=0,014 \% ; \mathrm{Fe}=0,35 \%$; Yodo $=0,001 \%$.

nera importante a la PB de los tratamientos; no obstante, debe tenerse presente que se encuentra asociada a la adición de taninos y su posible impacto sobre la eficiencia nutrimental de las dietas puede ser negativo ya que reduce drásticamente la degradación de proteínas y fibras en rumen especialmente de hemicelulosa (Frutos et al., 2004). Sin embargo, Velázquez et al. (2011) no encontraron diferencias significativas $(\mathrm{p}>0,05)$ al incluir hasta $400 \mathrm{~g} / \mathrm{kg}$ de $A$. farnesiana en dietas para ovinos.

Producción de gas y desaparición de la materia seca. En la tabla III se presentan los resultados de la PGIV y la figura 1 muestra la curva de producción de gas con respecto al tiempo. Se observa que los valores de 400 $\mathrm{g} / \mathrm{kg}$ de $A$. farnesiana son diferentes $(\mathrm{p}<0,05)$ al tratamiento control, posiblemente debido a la mayor concentración de TAN que impactó negativamente en la fermentación ruminal. Mientras que la tasa de fermentación $b\left(\mathrm{~h}^{-1}\right)$ y $T$ no presentaron diferencias entre tratamientos y la tasa de fermentación $c\left(\mathrm{~h}^{-1 / 2}\right) 300$ y $400 \mathrm{~g} / \mathrm{kg}$ de A. farnesiana fueron similares; pero, diferentes al control $(\mathrm{p}<0,05)$. Abdulrazak et al. (2000) reportaron una tasa de fermentación de $0,1165 \mathrm{~h}^{-1}$ en hojas de $A$. nubica y $0,0295 \mathrm{~h}^{-1}$ en $A$. brevispica los cuales son diferentes a los del presente estudio. Otros reportes en estudios similares coinciden en que una elevada concentración de TAN reducen la digestibilidad in vitro (Rubanza et al., 2005), aunque también debe considerarse la posible presencia de otros metabolitos secundarios como los aminoácidos no proteicos (ácido $\alpha$-acetyldiaminobutirico) encontrados en hojas de $A$. angustissima (McSweeney et al., 2005), atendiendo que su presencia puede tener un efecto sinérgico con los TAN (Makkar, 2003b; Salem y Gohar, 2004).

Los valores de DMSIV, PGR, y EMIV se muestran en la tabla IV. Se observa que la DMSIV y la PGR de 0 y $100 \mathrm{~g} / \mathrm{kg}$ de $A$. farnesiana fueron similares; pero mayores $(p<0,05)$ con respecto al resto de los tratamientos. Las figuras 2, 3 y $\mathbf{4}$ muestran las líneas de tendencia y la regresión de PGR, 
Tabla III. Efecto de la inclusión con diferentes niveles de inclusión $(\mathrm{g} / \mathrm{kg})$ de vainas de A. farnesiana sobre la producción de gas in vitro, utilizando la ecuación de France et al. (1993). (Effect of the inclusion with different levels $(\mathrm{g} / \mathrm{kg}$ ) of $A$. farnesiana pods on the in vitro gas production, using the equation proposed by France et al. (1993)).

\begin{tabular}{ccccc}
\hline Vainas de A. farnesiana & $A$ & $b$ & $c$ & $T$ \\
\hline 0 & $364,16^{\mathrm{d}}$ & $0,034^{\mathrm{d}}$ & $0,011^{\mathrm{d}}$ & $0,098^{\mathrm{d}}$ \\
100 & $364,92^{\mathrm{d}}$ & $0,035^{\mathrm{d}}$ & $0,019^{\mathrm{de}}$ & $0,048^{\mathrm{d}}$ \\
200 & $355,13^{\mathrm{d}}$ & $0,029^{\mathrm{d}}$ & $0,034^{\mathrm{ef}}$ & $0,013^{\mathrm{d}}$ \\
300 & $317,88^{\mathrm{de}}$ & $0,029^{\mathrm{d}}$ & $0,042^{\mathrm{d}}$ & $0,013^{\mathrm{d}}$ \\
400 & $304,45^{\mathrm{e}}$ & $0,027^{\mathrm{d}}$ & $0,043^{\mathrm{d}}$ & $0,009^{\mathrm{d}}$ \\
EEM & 0,865 & 0,011 & 0,018 & 0,038 \\
\hline
\end{tabular}

deDistintas literales en columnas presentan diferencias significativas $(p<0,05)$. $E E M=$ error estándar de la media; $A=$ producción total de gas $(\mathrm{ml} / \mathrm{g} \mathrm{MS}) ; b=$ tasa de fermentación $\left(\mathrm{h}^{-1}\right) ; c=$ tasa de fermentación $\left(\mathrm{h}^{-1 / 2}\right) ; T=$ tiempo lag $(\mathrm{h})$.

DMSIV y EMIV. Con respecto a la EMIV se observa que 300 y $400 \mathrm{~g} / \mathrm{kg}$ de inclusión de A. farnesiana fueron similares, aunque en relación al tratamiento control fueron menores $(\mathrm{p}<0,05)$; mientras que para TD no se observó diferencias ( $\mathrm{p}>0,05)$, entre los tratamientos que tuvieron un rango comprendido de $\mu 6=0,35$ a 0,39 . Estos resultados son afines a lo reportado por Rubanza et al.

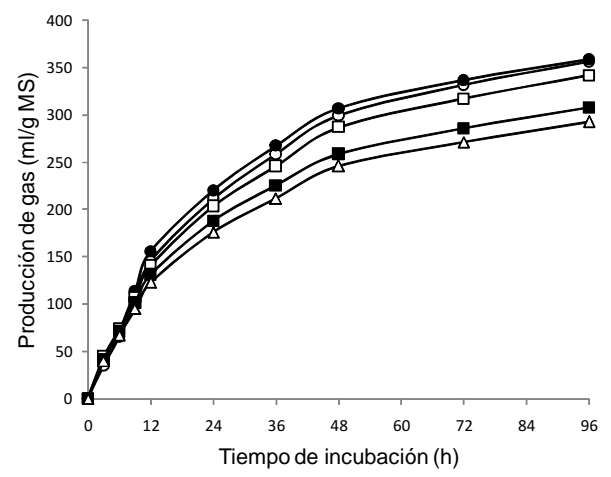

Figura 1. Producción de gas ( $\mathrm{ml}$ gas $/ \mathrm{g} M S$ ) durante la fermentación in vitro con diferentes niveles 0; 100; 200; 300; y $400 \mathrm{~g} / \mathrm{kg}$ de vainas de A. farnesiana. (Gas production (ml gas/g DM) during the in vitro fermentation with different levels $0 ; 100 ; 200 ; 300 ; y 400 \mathrm{~g} / \mathrm{kg}$ DM of A. farnesiana pods).
(2005), quien estudió el efecto de los TAN de hojas de Acacia sobre la digestibilidad in vitro usando polietilenglicol, indicando que la energía metabolizable aumentó conforme disminuyó la concentración de TAN. Una explicación posible al afecto de los tratamientos sobre la PGIV, DMSIV, PGR y EMIV encontrados en este estudio se relaciona con el nivel de inclusión de TAN, ya que su

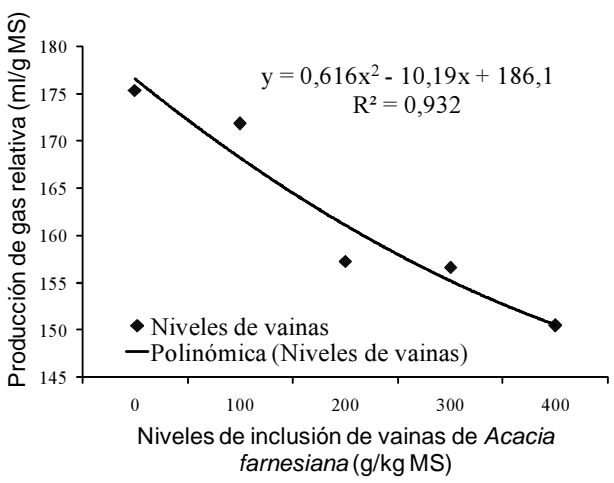

Figura 2. Producción de gas relativa $(\mathrm{ml}$ gas $/ g$ MS) durante la fermentación in vitro con diferentes niveles de vainas de A. farnesiana. (Relative gas production $(\mathrm{ml}$ gas $/ \mathrm{g}$ DM) during the in vitro fermentation with different levels of $A$. farnesiana pods). 
Tabla IV. Efecto de la inclusión ( $\mathrm{g} / \mathrm{kg} \mathrm{MS})$ de vainas de A. farnesiana en la desaparición de la materia seca in vitro producción de gas relativa y energía metabolizable. (Effect of the inclusion ( $/ \mathrm{kg} \mathrm{DM}$ ) of $A$. farnesiana pods on the in vitro dry matter disappeared, relative gas production and metabolizable energy).

\begin{tabular}{cccr}
\hline Inclusion, g/kg MS & DMS & PGR & EMIV \\
\hline 0 & $70,14 \pm 5,21^{\mathrm{a}}$ & $175,36 \pm 13,05^{\mathrm{a}}$ & $9,38 \pm 0,40^{\mathrm{a}}$ \\
100 & $68,73 \pm 1,74^{\mathrm{a}}$ & $171,83 \pm 4,35^{\mathrm{a}}$ & $8,92 \pm 0,38^{\mathrm{ab}}$ \\
200 & $62,92 \pm 2,39^{\mathrm{b}}$ & $157,30 \pm 6,00^{\mathrm{b}}$ & $9,07 \pm 0,22^{\mathrm{a}}$ \\
300 & $62,64 \pm 2,02^{\mathrm{b}}$ & $156,62 \pm 5,05^{\mathrm{b}}$ & $8,43 \pm 0,26^{\mathrm{bc}}$ \\
400 & $60,19 \pm 1,75^{\mathrm{b}}$ & $150,48 \pm 4,40^{\mathrm{b}}$ & $8,10 \pm 0,19^{\mathrm{c}}$ \\
EEM & 0,454 & 0,718 & 0,063 \\
\hline
\end{tabular}

${ }^{a b c}$ Distintas literales en columnas presentan diferencias significativas $(p<0,05)$. EEM= error estándar de la media; DMS= proporción de MS desaparecida (\%); PGR= producción de gas relativa ( $\mathrm{ml} \mathrm{gas/g} \mathrm{MSD);}$ $\mathrm{EMIV}=$ energía metabolizable in vitro (MJ/kg MS).

efecto positivo o negativo depende de su concentración en las dietas (Min et al., 2003), y al consiguiente efecto antimicrobiano y antifungal, que incluso, puede llegar a detener la actividad de fermentación si los niveles de concentración de TAN son superiores (Hervás et al., 2003; Salem et al., 2004).

Velázquez et al. (2011) no encontraron

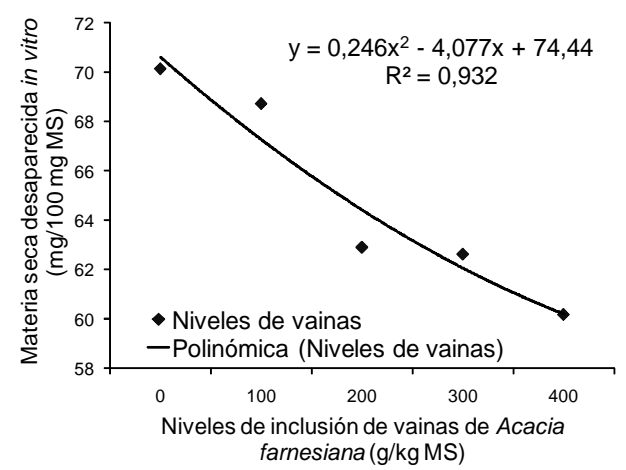

Figura 3. Análisis de regresión de la materia seca desaparecida in vitro con diferentes niveles de inclusión de vainas de A. farnesiana. (Regression analysis of the in vitro dry matter disappeared with different inclusion levels of $A$. farnesiana pods). diferencias significativas ( $p>0,05)$ en cuanto a la digestibilidad de la MS y FND en dietas para corderos con niveles crecientes de inclusion de Acacia farnesiana, hasta $400 \mathrm{~g} / \mathrm{kg} \mathrm{MS}$, lo cual podría indicar que efectivamente una concentración de $3,6 \mathrm{~g} / \mathrm{kg}$ MS de taninos no afecta los parámetros productivos en dietas para ovinos; sin embargo, a nivel ruminal este efecto se observa

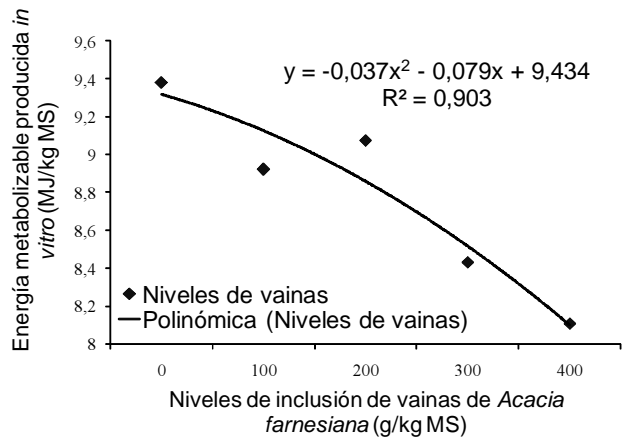

Figura 4. Producción de energía metabolizable (MJ/kg MS) durante la fermentación in vitro de vainas de $\mathrm{A}$. farnesiana con diferentes niveles de inclusión. (Metabolizable energy production $(\mathrm{MJ} / \mathrm{kg} \mathrm{DM})$ during the in vitro fermentation of $A$. farnesiana pods at different inclusion levels). 


\section{VELÁZQUEZ, GONZÁLEZ RONQUILLO, BÓRQUEZ, DOMÍNGUEZY PEREZGROVAS}

cuando se incluyen $200 \mathrm{~g} / \mathrm{kg}$ de Acacia farnesiana, ya que disminuye la DMSIV y PGR, afectando posiblemente la síntesis de proteína microbiana en el rumen, aunque no se ve afectado el comportamiento productivo de los animales (Velázquez et al., 2011), sí se ve afectada la EMIV cuando se incluyen $300 \mathrm{~g} / \mathrm{kg}$ de A. farnesiana.

\section{CONCLUSIONES}

Se concluye que la inclusión de $300 \mathrm{~g} / \mathrm{kg}$ MS de vainas de $A$. farnesiana impacta negativamente en la producción de gas y utilización de la energía metabolizable, y a

\section{BIBLIOGRAFÍA}

Abdulrazak, S.A., Fujihara, T., Ondiek, J.K. and Ørskov, E.R. 2000. Nutritive evaluation of some Acacia tree leaves from Kenya. Anim. Feed Sci. Technol., 85: 89-98.

Aganga, A., Adogla, B., Omphile, U.J. and Tshireletso, K. 2000. Significance of browses in the nutrition of Tswana goats. Arch. Zootec., 49: 469-480.

Aganga, A.A., Adogla, B. 1999. Dry matter degradation, tannin and crude protein contents of some indigenous browse plants of Botswana. Arch. Zootec., 48: 79-83.

Aganga, A., Tsopito, C.M. and Adogla, B. 1998. Feed potential of Accacia species to ruminants in Botswana. Arch. Zootec., 47: 659-668.

Arriaga-Jordán, C., Flores-Gallegos, F., PeñaCarmona, G., Albarrán-Portillo, B., GarcíaMartínez, A., Espinosa-Ortega, A., GonzálezEsquivel, C. and Castelán-Ortega, O. 2001. Participatory on-farm evaluation of the response to concentrate feeding supplementation by cows in early lactation in smallholder peasant dairy production systems in the highlands of central Mexico. J. Agric. Sci., 137: 97-103.

AOAC. 1990. Association of Official Analytical Chemists. $15^{\text {th }}$ ed. INC. VA. Official methods of analysis. Helrich. USA.

Benny, E., Tapia, B., Arroyo, J., Bermúdez, M., Espinosa, R. and Sánchez, F. 2001. In vitro ruminal digestibility of mesquite and huizache by effect of the tannin concentration. XVII Meeting of the Latin American Association of Animal partir de $200 \mathrm{~g} / \mathrm{kg}$ MS afecta la desaparición de la materia seca y producción de gas relativa in vitro lo cual podría limitar su uso y aprovechamiento para la alimentación de rumiantes. Se requieren estudios in vivo para poder comparar estos resultados.

\section{AGRADECIMIENTOS}

Este proyecto fue financiado parcialmente por el proyecto UAEMex 2200/2005 y fundación PRODUCE, ICAMEX folio 152005-1186. Jorge Antonio Velázquez Avendaño fue becado por CONACYT, México.

Production. La Habana. Cuba. Memories.

Ewin, W.N. 1997. The feeds directory. Commodity Products guide. Context Publications. Santa Rosa. CA.

France, J., Dhanoa, M.S., Theodorou, M.K., Lister, S.J., Davies, D.R. and Isaac, D. 1993. A model to interpret gas accumulation profiles associated with in vitro degradation of ruminant feeds. $J$. Theor. Biol., 163: 99-111.

Frutos, P., Hervás, G., Giradles, F.J. and Mantecón, R. 2004. Tannins and ruminant nutrition. J. Agric. Res., 2: 91-202.

González, R.M., Fondevila, M., Barrios, U.A. and Newman, Y. 1998. In vitro gas production from buffel grass (Cenchrus ciliaris L.) fermentation in relation to the cutting interval, the level of nitrogen fertilization and the season of growth. Anim. Feed Sci. Technol., 72: 19-35.

Hervás, G., Frutos P., Giráldez, F., Mantecón, A. and Álvarez del Pino, M. 2003. Effect of different doses of quebracho tannins extract on rumen fermentation in ewes. Anim. Feed Sci.Technol., 109: 65-78.

Makkar, H.P.S. 2003a. Quantification of tannins in tree and shrub foliage. Laboratory manual. $5^{\mathrm{a}}$ ed. Kluwer Academy Publisher. London. 101 pp.

Makkar, H.P.S. 2003b. Effects and fate of tannins in ruminant animals. Adaptation to tannins and strategies to overcome detrimental effects of feeding tannin-rich feeds. Small Rum. Res., 49: 241-256.

McSweeney, C.S., Palmer, B., McNeill, D.M. and 


\section{PRODUCCIÓN DE GAS IN VITRO CON INCLUSIÓN DE ACACIA FARNESIANA}

Krause, D.O. 2005. Microbial interactions with tannins: nutritional consequences for ruminants. Anim. Feed Sci. Technol., 91: 83-93.

McSweeney, C.S., Gough, J., Conlan, L.L., Hegarty, B.P. and Krause, D.O. 2005. Nutritive value assessment of the tropical shrub legume Acacia angustissima: anti-nutritional compounds and in vitro digestibility. Anim. Feed Sci. Technol., 106: 3-19.

Menke, K.H. and Steingass, H. 1988. Estimation of the energetic feed value obtained from chemical analysis and in vitro production using rumen fluid. Anim. Res. Devel., 28: 7-55.

Min, B.R., Barry, T.N., Attwood, G.T. and McNabb, W.C. 2003. The effect of condensed tannins on the nutrition and health of ruminants fed fresh temperate forages: Anim. Feed Sci. Technol., 106: 3-19.

Monforte-Briceño, G.E., Sandoval-Castro, C.A., Ramírez-Avilés, L. and Capetillo-Leal, C.M. 2005. Defaunating capacity of tropical fodder trees: effects of polyethylene glycol and its relationship to in vitro gas production. Anim. Feed Sci. Technol., 123: 313-327.

NRC. 2007. National Research Council. Nutrient requirements of small ruminants: sheep, goats, cervids, and new world camelids of National Research Council. Committee on Nutrient Requirements of Small Ruminants. US. 362 pp.

Parra, V.M.R. 1989. The agricultural subdevelopment in the highlands of Chiapas. Centro de Investigaciones Ecológicas del Sureste. Universidad Autónoma de Chapingo. Chiapas. Mexico.

Perezgrovas, G.R. 1999. The sheep of San Juan. Ovine indigenous system in the highlands of Chiapas. $2^{\mathrm{a}}$ ed. Instituto de Estudios IndígenasUNACH. Chiapas. México.

Purata, S., Greenberg, R., Barrientos, V. and López-Portillo, J. 1999. Economic potential of the huizache, A. pennatula (Mimosoideae) in Central Veracruz, México. Econ. Bot., 53: 1529.

Rubanza, C.D.K., Shem, M.N., Otsyna, R., Bakengesa, S.S., Ichinohe, T. and Fujihara,T. 2005. Polyphenolics and tannins effect on in vitro digestibility of selected Acacia species leaves. Anim. Feed Sci. Technol., 119: 129-142.

Salem, A.F.Z.M. and Gohar, Y.M. 2004. Anaerobic ruminal bacterial isolates from the rumen of different ruminant animals and their response to different levels of tannic acid. In: Habe. F. (Ed.). $55^{\text {th }}$ Annual Meeting of the EAAP. Bled. Slovenia. $265 \mathrm{pp}$.

Salem, A.Z.M., Gohar, Y.M., El-Adawy, M.M. and Salem, M.Z.M. 2004. Growth-inhibitory effect of some antinutritional factors extracted from Acacia saligna leaves on intestinal bacteria activity in sheep. $12^{\text {th }}$ Scientific Conference of the Egyptian Society of Animal Production. ESAP. Mansoura. Egypt. 41: 283-300.

Salem, A.Z.M. 2005. Impact of season of harvest on in vitro gas production and dry matter degradability of Acacia saligna leaves with inoculums from three ruminant species. Anim. Feed Sci. Technol., 123: 67-79.

Seigler, D.S. 2003. Phytochemistry of Acaciasensu lato. Proceedings of the Phytochemistry and Legume/Animal Interaction. Symposia held at the $4^{\text {th }}$ International Legume Conference in Canberra. Australia. Bioch. System. Ecol., 31: 845-873.

Steel, R.G.D. and Torrie, J.H. 1980. Principles and procedures of statistics. $2^{\mathrm{a}}$ ed. McGraw-Hill International. New York. USA.

Theodorou, M.K., Williams, B.A., Dhanoa, M.S., Mc Allan, A.B. and France, J. 1994. A simple gas production method using a pressure transducer to determine the fermentation kinetics of ruminant feeds. Anim. Feed Sci. Technol., 48: 185-197.

Van Soest, P.J. 1994. Nutritional ecology of ruminants. $2^{\mathrm{a}}$ ed. University Press Cornell. 476 pp.

Velázquez, A.J., Perezgrovas, G.R., Velasco, Z.M., Zaragoza, M.L., Rodríguez, G.G. 2005. Assessment of quebracho (Acacia farnesiana) pods utilization in wool-sheep feeding. Arch. Zootec., 54: 535-540.

Velázquez, A.J., González Ronquillo, M., Perezgrovas, R., Bórquez, J. y Domínguez, V. 2011. Producción, digestibilidad y rentabilidad en corderos de dietas con vainas de $A$. farnesiana. Arch. Zootec., 60: 479-488. 